\title{
UTILIDAD Y VIGENCIA DE LAS TIPOBIBLIOGRAFÍAS Y SU AUSENCIA EN EL MUNDO DIGITAL
}

Jon Zabala-Vázquez

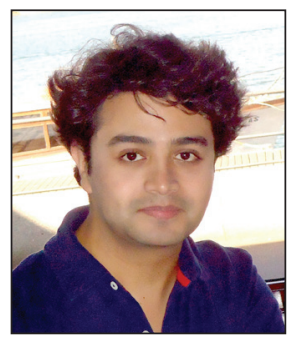

Jon Zabala-Vázquez es lingüista y bibliógrafo. Profesor en la Universidad Complutense de Madrid, por la que es doctor en ciencias de la información. Su docencia y líneas de investigación giran en torno a historia del libro, bibliografía material, fuentes de información, documentación audiovisual, lenguajes de marcado y tecnologías web.

http://orcid.org/0000-0002-7268-0821

Univ. Complutense de Madrid, Fac. de Ciencias de la Documentación Santísima Trinidad, 37. 28010 Madrid, España jzabala@pdi.ucm.es

\section{Resumen}

Se pretende mostrar la importancia y actualidad de los tiporepertorios para diversos estudios (textuales, ecdóticos, sociológicos, históricos, etc.). Dado que los trabajos del Proyecto Tipobibliografía Española (PTE) no están publicados en soporte digital, se reflexiona en torno a dicha necesidad, destacando la poca o nula utilidad de las principales normas y estándares automatizados de descripción para este propósito.

\section{Palabras clave}

Bibliografía material, Libro antiguo, Imprenta, Repertorios bibliográficos, Tipobibliografías, Proyecto Tipobibliografía Española, Catálogos automatizados, Humanidades digitales.

\section{Title: The utility and validity of bibliographies and their absence in the digital world}

\begin{abstract}
In this paper we analyze the importance and validity of bibliographies for various studies (textual criticism, sociological, historical, etc.). Because the Tipobibliografía Española project is not published in digital format, we emphasize that the main automated rules and standards of description are useless for this purpose.
\end{abstract}

\section{Keywords}

Critical bibliography, Descriptive bibliography, Analytical bibliography, Textual bibliography, Textual criticism, Ancient book, Early printed book, Hand-press book, Printing press, Bibliographies, Tipobibliografía Española Project, Online library catalogs, Digital humanities.

Zabala-Vázquez, Jon (2013). "Utilidad y vigencia de las tipobibliografías y su ausencia en el mundo digital". El profesional de la información, enero-febrero, v. 22, n. 1, pp. 68-73.

http://dx.doi.org/10.3145/epi.2013.ene.09

\section{Introducción}

En los últimos años se han digitalizado -en el marco de proyectos nacionales e internacionales por igual- centenares de colecciones de instituciones públicas y privadas, lo que, por su parte, se ha traducido en abundantes estudios sobre aspectos conceptuales, procedimentales y hasta deontológicos sobre la digitalización, los repositorios digitales, las aplicaciones multimedia e hipermedia, etc. De hecho, en esta misma revista, hace apenas unos números, se ponía de manifiesto la "necesidad de auxiliar a los académicos de humanidades en la creación, alojamiento, difusión y preservación de [...] recursos electrónicos" (Galina-Russell, 2012, p. 188).
Sin embargo, aunque la disposición de recursos electrónicos en la Red es un adelanto importante, es insuficiente. Estableciendo un paralelismo sinonímico con lo ocurrido hace unas décadas, así como un cúmulo de libros en un espacio físico no conformaban una biblioteca -si acaso un almacén-, hoy tampoco basta la mera disposición de miles de documentos electrónicos online. En la actualidad, como entonces, hacen falta herramientas (y servicios) documentales para convertir un conjunto de objetos inconexos en una verdadera fuente de información.

\section{Impresos del período manual}

Aunque la definición de "libro antiguo" es bastante ambigua, pues depende en buena medida de la perspectiva legal 


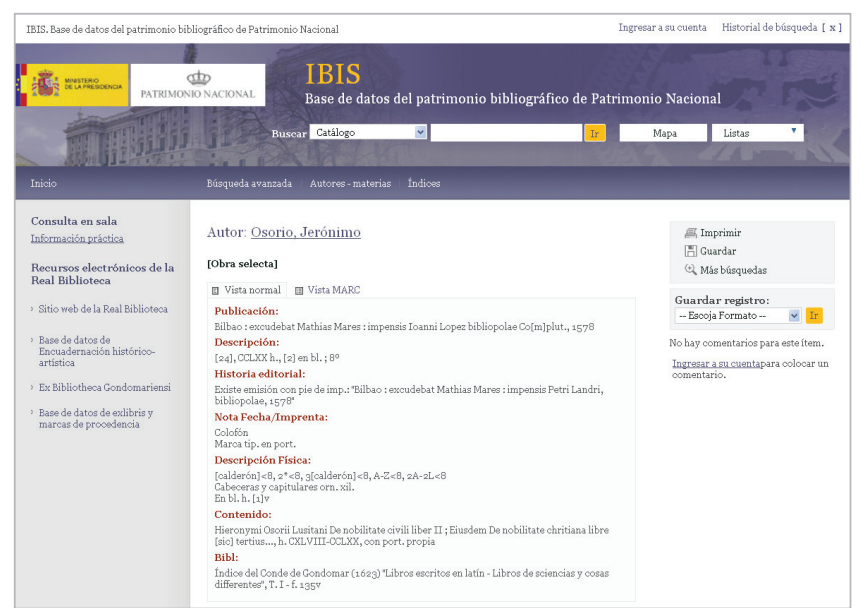

Figura 1. Registro catalográfico en IBIS (Base de datos del patrimonio bibliográfico de Patrimonio Nacional)

http://realbiblioteca.patrimonionacional.es

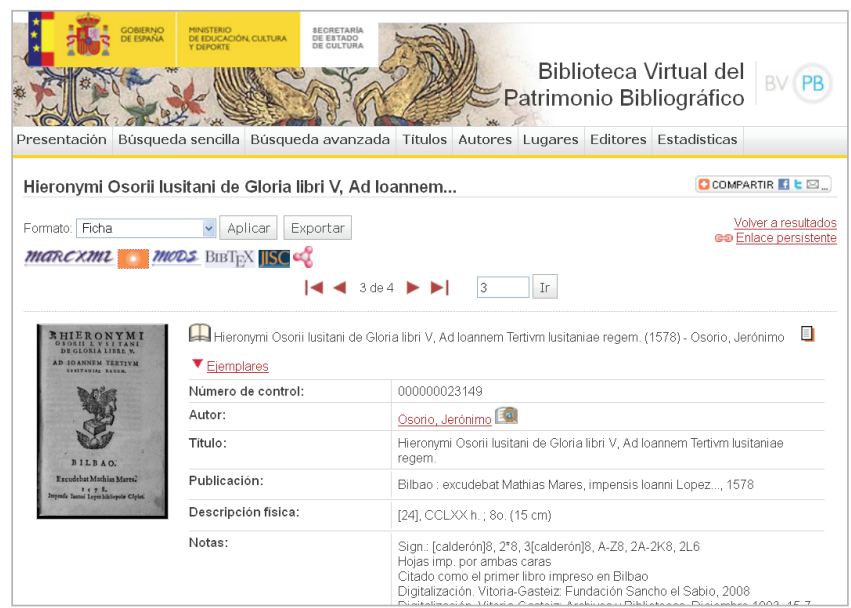

Figura 2. Registro catalográfico en la BVPB (Biblioteca virtual del patrimonio bibliográfico)

http://bvpb.mcu.es o normativa que se tenga en cuenta (De-los-Reyes-Gómez, 2003, pp. [11]-17), en la tradición anglosajona, donde se habla del hand-press period y del machine-press period, se diferencia con mayor claridad entre los libros impresos de forma artesanal -compuestos manualmente, impresos forma a forma y pliego a pliego, usando tintas y papel hechos a mano, etc. - y los producidos mecánicamente -fabricados mediante métodos industriales-. Los primeros, creados con anterioridad a 1820-1830, poseen características propias -importantes para la crítica textual y la ecdótica ${ }^{1}$ - que no siempre quedan al descubierto con el uso de los métodos modernos de análisis documental. Como sugirió Jaime Moll ([2011], pp. 12-13), introductor de la bibliografía material en España, es una quimera pensar que la imprenta facilitaba la reproducción de múltiples ejemplares idénticos, pues con los años se ha demostrado que la técnica empleada, justamente por su carácter artesanal, no lo permitía. En ese contexto, pues, los bibliógrafos anglosajones acuñaron las categorías conceptuales de edition (edición), issue (emisión) y state (estado), para diferenciar las manifestaciones físicas resultantes de aquel proceso de reproducción textual.

En el mundo digital se precisa de herramientas (y servicios) documentales para convertir el conjunto de objetos inconexos de la Red en una verdadera fuente de información

Pero además de esas diferencias surgidas en el momento mismo de su génesis, el paso del tiempo añadió otro problema al estudio de los impresos de ese período: el deterioro, la manipulación y la desaparición de algunos o todos sus ejemplares. A veces, como se puede suponer, por acciones directas como el expurgo o la destrucción por parte de las autoridades eclesiásticas o civiles de la época, por accidentes involuntarios -incendios, inundaciones u otras catástrofes-, o por la simple acción del paso del tiempo sobre las materias escriptorias - papel y pergamino, fundamentalmente-.
Y por citar un último inconveniente -aunque obviamente hay muchos más-, conviene recordar la dañina creación bibliográfica -intencionada o por omisión- de ediciones inexistentes. Basta una data mal interpretada -sobre todo en las formas de cómputo no convencionales-, un guarismo cambiado, una encuadernación defectuosa, la pertenencia a un volumen facticio, un nombre latino homónimo en la designación de un lugar, una transcripción incompleta o errónea... para aovar una edición imaginaria, de naturaleza fantasmal.

\section{El Proyecto Tipobibliografía Española (PTE)}

Por todo lo anterior, y siguiendo los principios de la New bibliography, surgida entre los estudiosos ingleses de finales del siglo XIX y principios del XX, en 1978 Frederick J. Norton publicó su magno $A$ descriptive catalogue of printing in Spain and Portugal 1501-1520. Éste, llamado a ser uno de los repertorios ibéricos de mayor importancia y utilidad de todos los tiempos, fue fuente de inspiración para el PTE, una iniciativa de talla nacional y participación mundial, consistente en "un inventario general y riguroso de toda la producción tipográfica nacional” (Simón-Díaz, 1991, p. 12), lo que la convirtió sin lugar a dudas en "el trabajo colectivo más importante realizado en el campo de nuestra bibliografía" (De-los-Reyes-Gómez, 2002, p. 171).

En su seno, a lo largo de tres lustros (1991-2005), se publicaron, amén de numerosos trabajos difundidos por otros medios, casi una docena de repertorios -en colaboración con la editorial Arco Libros-. En el primero, sobre la imprenta alcalaína del siglo XVI, Julián Martín-Abad, a manera de pauta y derrotero, sentó las bases de los trabajos posteriores, esto es: los objetivos básicos, la manera de alcanzarlos y la forma de presentar los resultados (las noticias). Y el último, de Mercedes Fernández-Valladares, supuso un salto cualitativo en el PTE por haber ofrecido un muestrario de las letrerías góticas utilizadas en los talleres burgaleses de la época, lo que le permitió identificar numerosos sine notis mediante un escrupuloso análisis tipográfico basado en el método Proctor-Haebler y los criterios establecidos por el propio Norton. Sin duda, el broche de oro de tan impor- 


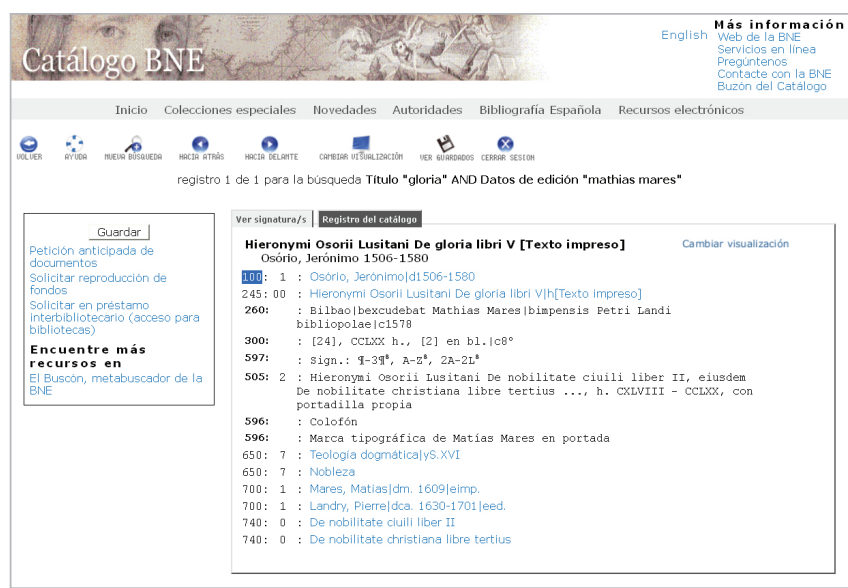

Registro catalográfico de la BNE (Biblioteca Nacional de España) http://catalogo.bne.es

tante y necesario proyecto bibliográfico y editorial, pues estos repertorios son los únicos que "permite[n] el (posible) control de la producción tipográfica antigua" (Martín-Abad, [2004], p. 133).

\section{Catálogos vs. bibliografías}

Aunque las tecnologías digitales y los nuevos soportes documentales han transformado, desdibujado o allanado ciertas fronteras conceptuales en muchas actividades cotidianas y/o académicas -antes consideradas inamovibles-, en algunos otros casos apenas han supuesto algún cambio.

La imprenta manual nunca posibilitó la reproducción de ejemplares idénticos, pues prácticamente todos tenían algún tipo de variante

Por ejemplo, un catálogo puede adoptar la forma de una base de datos, y lo mismo se puede hacer con un repertorio. Pero, a fuerza de insistir, los catálogos y las bibliografías son herramientas con objetivos claramente diferentes, aunque cercanos y afines por su naturaleza.

Por ello, volviendo a la realidad digital explicada en la introducción, aunque la publicación online de catálogos individuales y/o colectivos -regionales, nacionales o internacionales- y la digitalización masiva de ejemplares han facilitado significativamente la identificación y consulta de textos tipográficos antiguos, aún queda mucho por hacer. En ese sentido, aunque es fácil y frecuente escuchar que la elaboración de repertorios ha quedado desfasada por el desarrollo de los motores de búsqueda y la Red, o que los repositorios de objetos digitales han invalidado las representaciones mediante noticias, hay argumentos suficientes para rebatir tales opiniones -al menos en lo que al "libro antiguo" se refiere, por las singularidades que ya se han explicado en los epígrafes anteriores-.

Y es que en las tipobibliografías se busca describir el ejemplar ideal, la ideal copy, una reconstrucción histórica -casi hipotética-, lo cual sólo se consigue si se reúne y coteja el mayor número de ejemplares concretos de una determina-

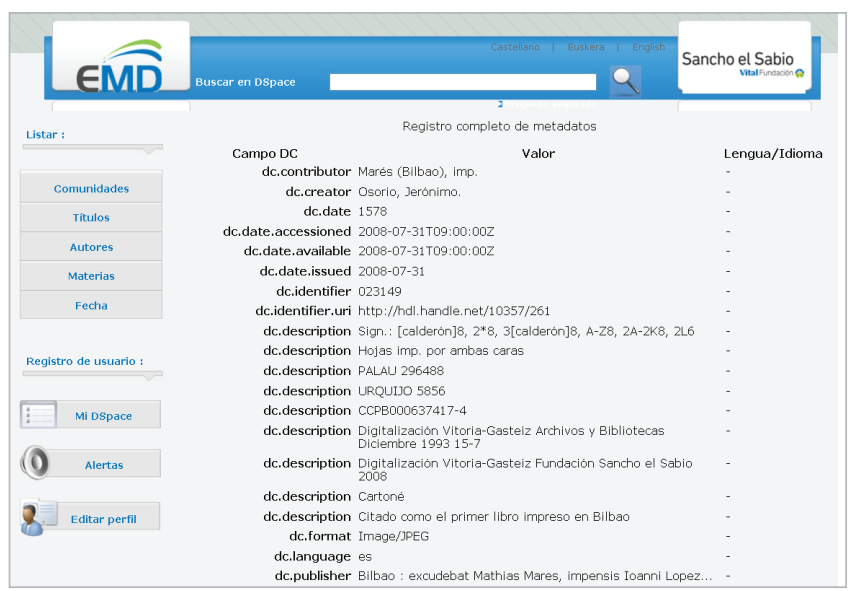

Figura 4. Utilización de la DCMI en EMD (Euskal memoria digitala) http://www.memoriadigitalvasca.es

da edición. Ejemplares que, como también ya se ha hecho notar, suelen estar dispersos geográficamente y poseen características individuales que son ajenas al proceso de edición, pero que también tienen su importancia al contar historias particulares de posesión o procedencia². Dicho de otra forma, un catálogo institucional -offline u online- suele estar compuesto por un conjunto de registros bibliográficos que se corresponden con objetos físicos precisos y, en el mejor de los casos, con una reproducción, posibilitando su consulta presencial -porque se incluye una signatura topográfica- o a distancia -por ofrecer una copia digital-. Mientras que, por otro lado, las bibliografías tipográficas dan cuenta de ediciones ideales (hipotéticas), deducidas mediante el examen de los vestigios conservados en forma de ejemplares concretos.

Aun así, los unos (catálogos) y las otras (tipobibliografías), a pesar de sus indudables diferencias en los objetivos y procedimientos, conviven en una beneficiosa simbiosis, como se evidencia, especialmente, en iniciativas colaborativas como el Catálogo colectivo del patrimonio bibliográfico español (CCPB) -con un cuarto de siglo de existencia-, donde las ideas de ejemplar ideal y emisión están cada vez más presentes. De hecho, en la práctica es cada vez más habitual que -para clarificar la existencia o ausencia de ediciones diferentes, o para establecer las emisiones de una edición concreta- los bibliotecarios incorporen en sus catálogos los resultados de los tipobibliógrafos, pues los primeros no pueden (ni deben) desplazarse físicamente para analizar in situ otros ejemplares, como, por el contrario, es de obligado cumplimiento para los segundos.

\section{Estructuras estandarizadas vs. noticias tipobibliográficas}

Explicadas las semejanzas y diferencias del epígrafe anterior, conviene enfatizar que las normas bibliotecarias de descripción están pensadas para representar "the item in hand as an exemplar of the entire manifestation \{el ejemplar que se tiene en la mano como un ejemplo de toda la manifestación\}" (ISBD, 2011, p. xii). Y en ese sentido, el formato $M A R C$, que hereda en parte las características de dichas normas, está también diseñado para estructurar y hacer legibles los registros bibliográficos de un catálogo informati- 
zado (figuras 1-3). Lo mismo se puede decir de otras iniciativas genéricas para la descripción de recursos electrónicos, como la DCMI (Dublin core metadata initiative) que, según el one-to-one principle, también se fundamenta en la descripción de manifestaciones bibliográficas concretas (fig. 4).

En ese contexto, pues, sin entrar en detalles innecesarios, es evidente que la estructura interna de un registro catalográfico -aun prescindiendo de sus puntos de acceso, sus signaturas topográficas y sus números de clasificación-, es muy distinta a la de una noticia tipobibliográfica (fig. 5), pues en esta última se procura -además de la identificación del ejemplar - la identificación tipográfica, editorial, textual, bibliográfica e histórica de cada texto materializado en el período antes señalado (Martín-Abad, 2003, pp. 181202; [2004], pp. [61], 81-85, [87]-88, [109]-110, [129]-133; [2001], pp. 20-21).

\section{Un catálogo nunca es una bibliografía retrospectiva, aunque muchos funjan como repertorios o se utilicen como ta- les (J. Martín-Abad)}

Para profundizar en los preceptos y métodos de la bibliografía material -más cercana a filólogos que a documentalistas-, no se pueden dejar de consultar los clásicos manuales de Fredson Bowers (1994), Ronald McKerrow (1994) y Jaime Moll ([2011]), y los repertorios de Norton (ibídem, pp. xx-xxiii), Martín-Abad (1991, pp. 43-45; [2001], pp. 15-28) y Fernández-Valladares (2005, pp. 116-125). De entre ellos, el último es particularmente útil para hacerse una idea bastante certera de cómo se hace una "buena" bibliografía de esta clase, cuyos elementos -algunos divisibles- se resumen en la fig. 6, donde se evidencian las diferencias existentes entre las noticias de una bibliografía por un lado, y los registros de un catálogo y la descripción de un recurso digital por el otro.

$1 \mathrm{~B}$

OSORIO, Jerónimo (Obispo de Sylves). De gloria libri $V$ [,] De nobilitate civili liber II [y] De nobilitate christiana liber I. Bilbao: Excudebat Mathias Mares: Impensis Petri Landri [...], 1578.

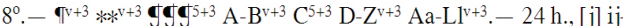
xvii [xviij-xx] xxj [xxij-xxiiij] xxv-xxvj [xxvij] xxviij-lvj [lvij-lxj] lxij [lxiij] lxiiij-lxxx [lxxxj] lxxxij-cxiiij [cxv] cxvj-cxxiij [cxxiii]] cxxvcxxxviij [cxxxix] cxl-clv [clvi] clvij-clxx [clxxj] clxxij-clxxxv [clxxxvij] clxxxviij [clxxxix] cxc-cxcviij [cxcix-ccj] ccij-ccxlix [ccl] cclj-cclx [cclxj] cclxij-cclxx f., 2 h. presumiblemente en blanco. -37 pl.- L. red.

Erratas en sign.: C - C 5 (en lugar de C - C v), H 3 (H iij), H 5 (H v), D v (V v).

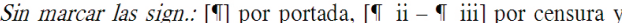

licencia, [** ij], [** v], [T iiij] por portada interior.

$[\ldots]$

[П] r: Portada:

* HIERONYMI | OSORII LVSITANI | DE GLORIA LIBRI. V. | AD IOANNEM TERTIVM | LVSITANIAE REGEM. | [Marca xilográfica de un grifo sobre un sillar unido a una bola alada. Similar a la utilizada por S. Gryphe (no. 211 y 870 de Silvestre), pero sin la leyenda] | BILBAO. | Excudebat Mathias Mares. | I578. | Impenfis Petri Landri, bibliopolæ.

[...]

Figura 5. Fragmento de una noticia tipobibliográfica
En dicha figura, además de las lógicas correspondencias y elementos comunes, queda patente que las noticias tipobibliográficas -metodológicamente pensadas para reflejar la reconstrucción de un ejemplar ideal-, poseen otros rasgos distintivos que, con los estándares bibliotecarios de descripción, sólo se pueden representar mediante la repetición sucesiva de notas generales y específicas, lo que obviamente reduce su capacidad expresiva y precisión semántica. Dicho de otra forma, dado que persiguen objetivos distintos, aunque no antagónicos, los estándares para la publicación de catálogos online apenas resultan útiles para la publicación electrónica de repertorios tipobibliográficos.

Como prueba de ello, aun en los casos de bases de datos con información sobre ejemplares ideales (?), como el Incunabula short title catalogue (ISTC)-donde se incluyen los datos generales de la obra, algunas referencias previas, notas de índole variada e indicaciones de ejemplares concretos (aunque sin signaturas topográficas)-, sus registros también carecen de la profundidad de análisis y de la exhaustiva descripción de las noticias tipobibliográficas, pues en última instancia no deja de ser un catálogo (fig. 7).

\section{Consideraciones finales}

Parece, pues, que los opacs y los millares de documentos digitalizados -aunque útiles- son insuficientes para la realización de los estudios ecdóticos y textuales, porque se sigue dejando a los investigadores la ardua y dilatada tarea de buscar, identificar, analizar, cotejar, discriminar y clasificar esos ejemplares individuales para, sólo entonces, dedicarse a la labor filológica. Lo que es más, la mayor parte de esas copias digitales han sido escaneadas sin tener en cuenta los principios de la bibliografía material, por lo que es bastante habitual encontrarse con recursos digitales sin ninguna clase de referencias métricas o cromáticas que permitan analizarlos con precisión, donde el estándar "tamaño pantalla" no siempre permite distinguir entre un octavo y un dozavo -por poner un ejemplo-, pues todos son iguales en apariencia. Eso sin mencionar los peligrosos pero apetecibles retoques, (re)composiciones y demás modificaciones digitales que pueden alterar la percepción que se tenga del original.

\section{Los repertorios tipobibliográficos son los únicos que permiten el verdadero con- trol de la producción tipográfica antigua de una localidad (J. Martín-Abad)}

Dicho esto, la labor del bibliógrafo en la compilación de tipobibliografías sigue siendo tan útil y necesaria como en los comienzos del PTE, cuyas formulaciones se mantienen vigentes tres décadas después de su puesta en marcha. Por ello, en la era digital, es cada vez más apremiante que estos repertorios se publiquen en un soporte informático, lo que favorecería no sólo su visibilidad y difusión, sino también su uso, al permitir búsquedas -generales y específicas- en todo su corpus, más allá de los puntos de acceso e índices preparados por el propio tipobibliógrafo.

Sin embargo, queda por delante el reto de preparar una estructura informática hecha a medida para las tipobiblio- 


\begin{tabular}{|c|c|c|c|c|c|}
\hline \multicolumn{3}{|c|}{ Noticia tipobibliográfica } & AACR2, ISBD (A) & MARC & DCMI \\
\hline \multirow{7}{*}{$\begin{array}{l}\text { Noticia sintética } \\
\text { (referencia) }\end{array}$} & \multicolumn{2}{|l|}{ autoridades } & [título] y mención de responsabilidad & 100,110 & creator \\
\hline & \multicolumn{2}{|l|}{ título } & título [y mención de responsabilidad] & 245,240 , et al. & title \\
\hline & \multicolumn{2}{|c|}{ responsabilidades secundarias } & [título] y mención de responsabilidad & 700 & contributor \\
\hline & \multirow{4}{*}{$\begin{array}{l}\text { publicación y } \\
\text { distribución }\end{array}$} & lugar & \multirow{4}{*}{ publicación, impresión o distribución } & $260 \$ a, 752$ & \\
\hline & & editor & & $260 \$ b$ & publisher \\
\hline & & año & & $260 \$ c$ & date \\
\hline & & colofón & & 260,596 & \\
\hline \multirow{7}{*}{ Descripción física } & \multicolumn{2}{|l|}{ formato } & descripción física & 300 & description \\
\hline & \multicolumn{2}{|l|}{ colación } & notas: descripción física & 597 & description \\
\hline & \multicolumn{2}{|l|}{ extensión } & descripción física & 300 & description \\
\hline & \multicolumn{2}{|l|}{ tipo de letra } & notas: descripción física & 597 & description \\
\hline & \multicolumn{2}{|l|}{ [pliegos*] } & descripción física; notas: descripción física & 300,597 & description \\
\hline & \multicolumn{2}{|l|}{ erratas } & notas: descripción física & 597 & description \\
\hline & \multicolumn{2}{|c|}{ disposición textual } & notas: relativas al contenido & 505 & description \\
\hline \multirow{4}{*}{$\begin{array}{l}\text { Transcripción } \\
\text { (contenido) }\end{array}$} & \multicolumn{2}{|c|}{$\begin{array}{l}\text { paratextos (portadas, portadillas, } \\
\text { índices, sumarios, licencias, privilegios, } \\
\text { aprobaciones o censuras, colofones, } \\
\text { tasas, dedicatorias, etc. }\end{array}$} & notas: relativas al contenido & 500,505 & description \\
\hline & \multicolumn{2}{|l|}{ textos } & notas: relativas al contenido & 505 & description \\
\hline & \multicolumn{2}{|l|}{ [lengua*] } & notas: lengua & 546 & language \\
\hline & \multicolumn{2}{|l|}{ [tema*] } & & 650 & subject \\
\hline $\begin{array}{l}\text { Tradición } \\
\text { bibliográfica }\end{array}$ & \multicolumn{2}{|c|}{ referencias previas } & notas: referencia bibliográfica & $504,510,595$ et al. & relation \\
\hline \multirow[t]{2}{*}{ Ejemplares } & \multicolumn{2}{|c|}{$\begin{array}{l}\text { lugares, instituciones, signaturas } \\
\text { topográficas }\end{array}$} & $\begin{array}{l}\text { notas: encuadernación y condiciones de } \\
\text { adquisición; relativas al ejemplar }\end{array}$ & $\begin{array}{l}563,901,903,752, \\
\text { et al. }\end{array}$ & \\
\hline & \multicolumn{2}{|l|}{ no aplican } & $\begin{array}{l}\text { identificador tipográfico (en otras ISBD: } \\
\text { números normalizados) }\end{array}$ & & $\begin{array}{l}\text { coverage } \\
\text { format } \\
\text { identifier } \\
\text { rights } \\
\text { source } \\
\text { type }\end{array}$ \\
\hline
\end{tabular}

Figura 6. Noticia tipobibliográfica vs. referencia catalográfica y una descripción con metadatos genéricos

grafías, pues, como se ha (de)mostrado en este artículo, los estándares para la comunicación e intercambio de registros -como el formato MARC- están pensados para eso, para contener, en forma de campos, la información de un registro catalográfico - con sus puntos de acceso y encabezamientos incluidos-. Campos que, al igual que otras iniciativas de marcado -como la DCMI-, resultan demasiado genéricos y redundantes para la delimitación semántica $-y$ la ordenación sintáctica- de los distintos elementos de una noticia tipobibliográfica.

\section{Los estándares para opacs apenas resul- tan útiles para la publicación online de repertorios tipobibliográficos}

Tal vez, de hecho, sólo un impulso desde las trincheras de lo electrónico, lo automatizado y lo digital pueda reavivar el PTE, cuya pretensión de completar el inventario general y riguroso de toda la producción tipográfica nacional está aún por alcanzar. Parafraseando a un buen filólogo, con ayuda de las TIC, las humanidades científicas pueden hoy recupe- rar el protagonismo perdido y "ser vanguardia y fuente de progreso" de la Sociedad que les da sentido (Lucía-Megías, [2012], p. 14).

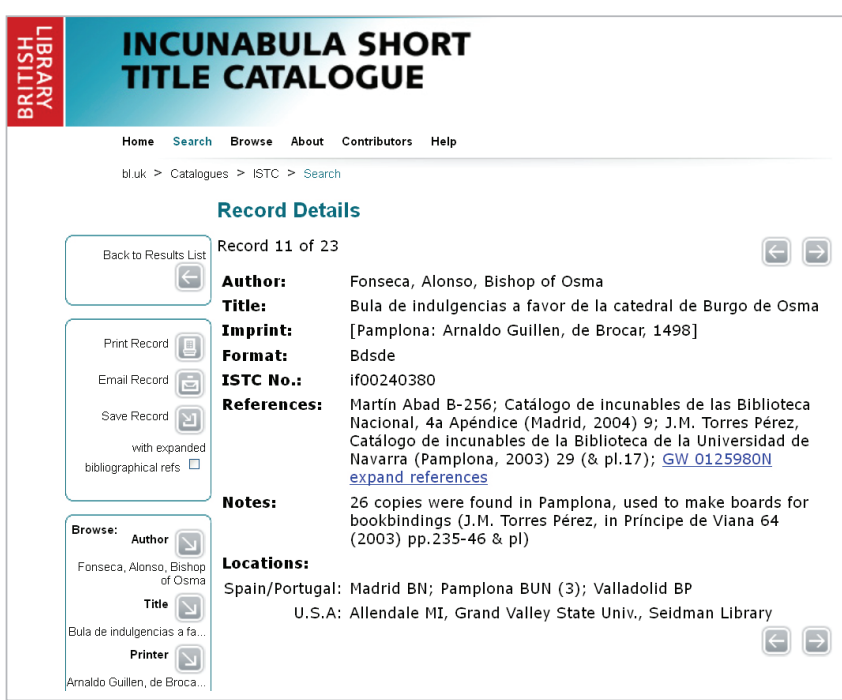

Figura 7. Registro catalográfico en el ISTC (Incunabula short title catalogue) http://www.bl.uk/catalogues/istc 


\section{Notas}

1. Se denomina ecdótica a la disciplina que se plantea el estudio de la totalidad de los elementos que conforman -o han de conformar- la edición de un texto, sin importar que éste sea manuscrito, impreso o digital (multimedia). Por tanto, va más allá de la crítica textual.

2. Aunque tradicionalmente en algunas bibliografías así se ha hecho, la inclusión de información relevante sobre la "vida" de cada ejemplar es un aspecto innovador que está empezando a calar hondo en muchos repertorios y catálogos, como bien se ilustra en el Bodleian Incunable Catalogue.

\section{Bibliografía}

Bowers, Fredson (1994). Principles of bibliographical description. Winchester: St. Paul's Bibliographies [et al.]. ISBN: 1884718000

De-los-Reyes-Gómez, Fermín (2002). "El proyecto «Tipobibliografía española»". Boletín de la Biblioteca de Menéndez Pelayo, año LXXVIII, pp. 171-197.

De-los-Reyes-Gómez, Fermín (2003). “Introducción”. En: Pedraza-Gracia, Manuel-José [et al.]. El libro antiguo. Madrid: Síntesis, pp. [11]-44. ISBN: 8497561538

Fernández-Valladares, Mercedes (2005). La imprenta en Burgos (1501-1600). Madrid: Arco Libros. ISBN: 8476356277.

Galina-Russell, Isabel (2012). "Retos para la elaboración de recursos digitales en humanidades". El profesional de la información, marzo-abril, v. 21, n. 2, pp. 185-189.

http://dx.doi.org/10.3145/epi.2012.mar.09
ISBD: International standard bibliographic description: [consolidated ed.] (2011). Berlin; Boston: De Gruyter Saur. ISBN: 9783110263794

Lucía-Megías, José-Manuel (2012). Elogio del texto digital: claves para interpretar el cambio de paradigma. Madrid: Fórcola. ISBN: 9788415174301

Martín-Abad, Julián (2001). Post-incunables ibéricos. Madrid: Ollero y Ramos. ISBN: 8478951652

Martín-Abad, Julián (2003). Los primeros tiempos de la imprenta en España (c. 1471-1520). Madrid: Laberinto. ISBN: 8484830861

Martín-Abad, Julián (2004). La imprenta en Alcalá de Henares (1502-1600). Madrid: Arco Libros. ISBN: 8476350953

Martín-Abad, Julián (2004). Los libros impresos antiguos. Valladolid: Universidad, Secretariado de Publicaciones e Intercambio Editorial. ISBN: 8484482790

McKerrow, Ronald. An introduction to bibliography for literary students. Winchester: St. Paul's Bibliographies [et al.], 1994. ISBN 1884718019

Moll, Jaime (2011). Problemas bibliográficos del siglo de oro. Madrid: Arco Libros, pp. [11]-78. ISBN: 9788476358160

Norton, Frederick J. (1978). A descriptive catalogue of printing in Spain and Portugal: 1501-1520. Cambridge [et al.]: Cambridge University Press.

Simón-Díaz, José (1991). “Introducción a la «Tipobibliografía española»". En: Martín-Abad, Julián. La imprenta en Alcalá de Henares (1502-1600). Madrid: Arco Libros, v. 1, pp. [7]-15. ISBN: 8476350961

\section{Próximos temas centrales}

Marzo 2013

Mayo 2013

Julio 2013

Septiembre 2013

Noviembre 2013

Enero 2014

Marzo 2014

Mayo 2014
Educación y biblioteca

Bibliotecas y documentación de museos

Economía de la información

Gestión de contenidos

Formación y aprendizaje

Políticas de información

Humanidades digitales

Big data y analítica web

Los interesados por favor consulten detenidamente las Normas para autores: http://www.elprofesionaldelainformacion.com/autores.html

y luego envíen sus artículos a través del gestor de manuscritos OJS de la plataforma del Recyt: http://recyt.fecyt.es/index.php/EPI/index 